\title{
Laboreal
}

Volume $9 \mathrm{~N}^{\circ} 1$ | 2013

Varia

\section{Un enfoque diacrónico de los TME : uso de datos cuantitativos en una gran empresa}

Uma abordagem diacrónica das LME : o uso de dados quantitativos numa grande empresa

Une approche diachronique des TMS : usage de données quantitatives dans une grande entreprise

A diachronic approach of MSD : use of quantitative data in a large company

\section{Céline Mardon, Willy Buchmann y Serge Volkoff}

Traductor. Céline Mardon

\section{OpenEdition}

\section{Journals}

Edición electrónica

URL: http://journals.openedition.org/laboreal/6085

DOI: 10.4000/laboreal.6085

ISSN: 1646-5237

Editor

Universidade do Porto

Referencia electrónica

Céline Mardon, Willy Buchmann y Serge Volkoff, « Un enfoque diacrónico de los TME : uso de datos cuantitativos en una gran empresa », Laboreal [En línea], Volume $9 N^{0} 1$ | 2013, Publicado el 01 julio 2013, consultado el 05 octubre 2019. URL : http://journals.openedition.org/laboreal/6085 ; DOI : $10.4000 /$ laboreal. 6085

Este documento fue generado automáticamente el 5 octubre 2019.

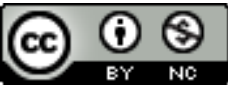

Laboreal está licenciado com uma Licença Creative Commons - Atribuição-NãoComercial 4.0 Internacional. 


\section{Un enfoque diacrónico de los TME : uso de datos cuantitativos en una gran empresa}

Uma abordagem diacrónica das LME : o uso de dados quantitativos numa grande empresa

Une approche diachronique des TMS : usage de données quantitatives dans une grande entreprise

A diachronic approach of MSD : use of quantitative data in a large company

Céline Mardon, Willy Buchmann y Serge Volkoff

Tradución : Céline Mardon

\section{NOTA DEL EDITOR}

Manuscrito recebido em : fevereiro/2013

Aceite após peritagem : maio/2013

\section{Introducción}

1 En esos últimos años, se han cumplido inmensos progresos en la comprensión y en la prevención de los mecanismos de aparición de los dolores articulares y de los trastornos músculo esqueléticos (TME) relacionados con el trabajo. Sin embargo, la realización de investigaciones con el objetivo de proseguir este esfuerzo de comprensión está todavía social y científicamente justificada, a pesar de que, como lo apuntan Aptel y Vézina (2008), el progreso de los conocimientos no implica forzosamente progresos en la acción :

2 (...) se impone el admitir que esto no implica sin embargo una voluntad colectiva efectiva de acción a la altura de la realidad del riesgo. Más aún, se asiste a un desajuste 
entre el nivel de conocimientos sobre el tema y el compromiso de los responsables de la prevención. Todo parece ocurrir como si los TME representaran un hándicap socialmente aceptado (p.2).

3 En este contexto, el cómputo de personas afectadas por esas patologías, así como más ampliamente por enfermedades laborales (EL), representa un asunto delicado pues manifiesta cuestiones sociales y económicas susceptibles de influir en el funcionamiento de los sistemas que las gestionan. El censo de los TME no escapa a estas cuestiones, puesto que estas patologías constituyen en la Unión Europea el primer motivo de enfermedad laboral (Eurogip, 2007). Por otro lado, toda comparación europea es relativa y las condiciones jurídicas nacionales de subsanación pesan sobre las estadísticas de EL (Eurogip, 2007). En relación a Francia, en 2010, las EL relativas a las afecciones peri-articulares del miembro superior representaban cerca del $80 \%$ del total de las EL reconocidas. Pero recientes estudios incitan a no considerar la cantidad de TME reconocidos como representativa de la amplitud del fenómeno (Ha \& Roquelaure, 2010). Estas cifras enmascaran toda la historia de los asalariados, desde las primicias del dolor hasta el proceso administrativo de declaración e incluso de reconocimiento de la enfermedad. Estas cifras representan un indicador muy restrictivo para evaluar con detalle la amplitud del fenómeno TME debido a las infradeclaraciones (trabajadores cuyo estado de salud osteo-articular podría caber en el ámbito reglamentario de subsanación, pero que por diversas razones no entablan el procedimiento administrativo de declaración de enfermedad laboral) y de las "nodeclaraciones" (trabajadores que presentan una patología osteo-articular relacionada con el trabajo pero que no caben en el ámbito de los cuadros de la CNAM [1]) (Diricq, 2011). De este modo sería conveniente interesarse en otros indicadores, para describir con más precisión la epidemia de TME a escala del conjunto de la población activa.

4 Según el nivel jerárquico en la empresa - del operador con dolores articulares, a la dirección de la empresa, pasando por el colectivo de trabajo o la jerarquía de proximidad - las preocupaciones asociadas a la gestión de los TME difieren. A pesar de ésto, todo el mundo se pone de acuerdo en decir que, de diferentes maneras, los TME representan un problema costoso (Fauconnier, Pépin \& Douillet, 2005). En este contexto, el envejecimiento del conjunto de la población activa en los países industrializados (Volkoff, 2012), con el impacto incierto de las políticas de prolongación de la vida laboral, el anhelo de un despido precoz por jubilación de los asalariados con mala salud , y también en relación al trabajo, la persistencia de exigencias físicas o la intensificación del trabajo (Askenazy, Cartron, De Coninck \& Gollac, 2006) nos incitan a colocar las condiciones de trabajo y la salud como base de los debates científicos y sociales sobre esos asuntos, y a adoptar una lectura diacrónica de los hechos estudiados a través de un enfoque combinado de las relaciones salud-trabajo, a lo largo de los recorridos profesionales.

5 Las teorías de la salud y de la salud en el trabajo, las contribuciones epidemiológicas y los estudios de medicina del trabajo incitan a insistir en el interés de tomar en cuenta la imbricación de varias dimensiones temporales, de medio y largo plazo (Plouvier, Gourmelen, Chastang, Lanoë \& Leclerc, 2011 ; Aptel \& Vézina, 2008).

6 Completar el análisis in situ de las situaciones laborales a través de un enfoque diacrónico constituiría entonces un aporte a la prevención (Cole, Manno, Beaton, \& Swift, 2002; Buchmann, Volkoff, \& Archambault, 2011). Los TME resultan de condiciones de exposición complejas cuyas características se elaboran a lo largo de los 
cambios sucesivos (evoluciones de las técnicas de producción, de evoluciones organizacionales, o también de evoluciones de las competencias, de los colectivos de trabajo, etc.). Un examen que cruce las evoluciones de tipo estructural con la llegada de señales precursoras sobre la salud puede dar nuevas claves de interpretación. Es importante tomar en cuenta estas señales precursoras, como los dolores considerados posteriormente en este artículo, ya que los TME comprenden episodios agudos que pueden volverse crónicos (Aptel \& Vézina, 2008), y que no presentan una remisión temporal clara (Aublet-Cuvelier, Aptel, \& Weber 2006 ; Silverstein et al. 2006). A través de un enfoque comprensivo que pueda tomar en cuenta el tiempo transcurrido a medio y largo plazo (Berg, Sanden, \& Torell, 1988), se trata entonces de seguir desenmarañando la madeja de las relaciones entre el trabajo y la salud.

7 En nuestra investigación, articulando enfoques cualitativos y cuantitativos (Mergler, 1999) y varios niveles (del micro en el puesto de trabajo, al macro a escala del grupo industrial), procuramos cotejar puntos de vista de varias disciplinas haciendo dialogar ergonomía, estadística, o incluso medicina del trabajo, con la diacronía como hilo conductor. Esta publicación no tiene el objetivo de rendir cuenta de la totalidad de este trabajo que, en el momento en que se escribe el presente texto, es objeto de una tesis doctoral en ergonomía. Se trata aquí de presentar los resultados de la parte cuantitativa de esta investigación.

\section{Un enfoque estadístico en un grupo aeronáutico}

8 El establecimiento que constituía el terreno de investigación pertenece a un gran grupo industrial francés, cuya medicina del trabajo se ha dotado de un observatorio cuantitativo plurianual que funciona desde 2002: el dispositivo Evrest (Evolutions et relations en santé travail [2]), que desde entonces fue adaptado a un nivel nacional e inter-empresas (Molinié \& Leroyer, 2011). Este observatorio fue construido en colaboración con médicos del trabajo e investigadores, con el objetivo de poder analizar y seguir en el tiempo varios aspectos del trabajo y de la salud de los asalariados. Fue concebido para durar, y por eso es forzosamente más elemental y "generalista" que una encuesta puntual acerca de un problema en particular. Se trata de un dispositivo arraigado en la práctica de los médicos y llevado por ellos.

9 La creación en esta empresa del dispositivo aspiraba a responder a dos objetivos principales : producir "indicadores" simultáneos sobre el trabajo y la salud, y sobre su evolución, haciendo además eco a las preocupaciones de la ergonomía de la actividad.

De este modo este observatorio se apoya en un cuestionario [3] corto (anverso y reverso) rellenado por un asalariado en cada visita médica sistemática (en esta empresa, esas visitas son espaciadas de uno a dos años, según el oficio de los asalariados). Este cuestionario comporta, además de los datos administrativos del asalariado, preguntas sobre sus condiciones de trabajo (horarios, presión temporal, apreciaciones sobre el trabajo, cargas físicas), viajes profesionales, formación, modo de vida, y una parte final rellenada por el médico del trabajo con informaciones bastante generales sobre el estado de salud del asalariado (especialmente cardiorrespiratorio, neuro-psíquico, y lo que nos importa aquí, osteo-articular).

11 Los problemas de TME eran una preocupación importante para los médicos del trabajo de ciertos establecimientos del grupo. Lo que constataban en su actividad médica fue apoyado por resultados cuantitativos producidos por Evrest (Buchmann, Mardon, 
Archambault, \& Volkoff, 2009), ya que más globalmente en la empresa las prevalencias de los problemas osteo-articulares son elevadas, prácticamente similares a las del nivel nacional, mientras que en relación al resto de problemas de salud, la situación en esta empresa parece ser netamente mejor que la media nacional. Por una parte, el personal del taller es el que presenta una prevalencia más elevada, y por otra parte, esta prevalencia se ve incrementada con la edad. En relación a las condiciones de trabajo según la edad, se constataba un ligero fenómeno de selección que protegía los operadores más mayores, pero esta muy ligera tendencia podía llevarnos a pensar que no había tantas reasignaciones posibles para los operadores [ $\left.{ }^{4}\right]$ que tienen problemas osteo-articulares. Estos resultados numéricos, que sugieren problemas de TME importantes y estables en el tiempo, constituían un argumento suplementario para afirmar que los problemas de salud osteo-articulares constituían una prioridad.

En el ámbito de esta investigación, Evrest ha contribuido a analizar de manera más completa las relaciones a lo largo del tiempo entre el trabajo y la salud osteo-articular, intentando considerar el estado de los dolores, no solo en un momento dado sino en un continuum, lo que se puede acercar a una preocupación más general : el debate social sobre la salud en el trabajo se refiere a menudo a fenómenos que merecen ser analizados según enfoques diacrónicos a medio y largo plazo (Volkoff \& Molinié, 2011). Porque a pesar de ser considerados a priori como dependientes de hechos inmediatos, se inscriben en la doble historia de los sistemas de producción y de las trayectorias profesionales. La lectura diacrónica de las relaciones entre el trabajo y la salud, que revisten varias formas, permite (con modelos e instrumentos adaptados) desembrollar las diversas dimensiones temporales implicadas en estos fenómenos.

Teniendo en cuenta el tema de esta investigación y la entrada diacrónica que incorpora naturalmente las cuestiones de edad, tres temas de investigación serán presentados sucesivamente, utilizando un desglose inspirado en las investigaciones sobre el envejecimiento. Efectivamente, los textos en ergonomía que tratan los fenómenos de envejecimiento en el trabajo sugieren distinguir tres procesos : fenómenos de desgaste (envejecimiento "por" el trabajo), fenómenos de selección (envejecimiento "con respecto" al trabajo) y fenómenos de regulación (envejecimiento "en" el trabajo) (Volkoff \& Gaudart, 2006). Nuestra investigación no se centra en el envejecimiento pero hemos elegido guardar este desglose, sustituyendo el análisis del envejecimiento por el análisis de los trastornos osteo-articulares, y adoptando un orden ligeramente diferente :

- Trataremos las regulaciones en el trabajo en primer lugar, justamente porque, si son eficientes, son susceptibles de proteger del desgaste y de la selección. Así, las relaciones salud-trabajo serán primero analizadas desde el punto de vista de los procesos de regulación, tratando de determinar si a un momento dado del itinerario profesional ciertos aspectos del trabajo de los operadores permiten (o no) limitar los efectos deletéreos conocidos de los factores físicos sobre los dolores.

- Después trataremos los fenómenos de desgaste y de cúmulo ya que remiten a huellas del pasado, cuando la regulación no es suficientemente protectora. La salud actual se pondrá entonces en relación con "trayectorias de esfuerzo" [5] en relación a los factores de riesgo TME, con el fin de poner de relieve los mecanismos de desgaste.

- Trataremos en un tercer tiempo los mecanismos de selección o de protección relacionados con los trastornos articulares, generalmente en acción cuando las regulaciones han fallado o siguen siendo insuficientes, y cuando el desgaste se vuelve demasiado importante para 
aguantar el trabajo. Esta tercera parte estará entonces dedicada al análisis de estos mecanismos de selección en acción a lo largo del tiempo, considerando las variaciones en el tiempo del "esfuerzo" [ $\left.{ }^{6}\right]$ físico, relacionado con el estado de salud en un punto inicial.

La batería de datos Evrest a nuestra disposición ha sido estudiada con técnicas diferentes y por poblaciones distintas según el tema investigado. Para facilitar la lectura, indicaremos por cada uno de estos tres enfoques, a qué ámbito conceptual en particular nos referimos, qué análisis realizamos (métodos y modos de cálculo utilizados, a partir del instrumento común ya presentado : el observatorio Evrest), qué resultados obtenemos, y qué comentarios estos resultados suscitan a nuestro parecer. Una discusión más general agrupando los tres enfoques será presentada al final del artículo.

Los software utilizados son Excel et SAS.

\section{Los mecanismos de "regulación" : ¿permiten los márgenes de maniobra limitar la sobrevenida de los dolores, o de mejor hacerles frente?}

\subsection{Repasos conceptuales}

Para introducir aquí la idea de regulación, podemos referirnos a Vézina (2001) :

la persona por su actividad tendrá que adaptarse y ajustarse continuamente. Así, desarrollará diferentes maneras de proceder o estrategias que le permitirán tomar en cuenta la variabilidad de las condiciones de trabajo y su propia variabilidad con el fin de mantener un equilibrio entre su salud y su producción. Cuando este proceso de regulación falla, la salud puede verse afectada (p.46).

17 Por consiguiente, unos márgenes de maniobra reducidos pueden conducir al desarrollo de TME. Existen entonces diferentes modalidades de regulación que los operadores construyen y ponen en acción para responder a las exigencias de la tarea a pesar de los dolores articulares. Laville y Volkoff (2004) proponen una categorización según la cual se reconocen dos principales modalidades de regulación, la regulación por evitación (que puede ser individual o por repartición de tareas en un colectivo) y la regulación por compensación, que se traduce en el desarrollo de regulaciones funcionales, posible únicamente si la organización y los medios de trabajo permiten márgenes de maniobra individuales y colectivos suficientes (márgenes temporales especialmente).

\subsection{Método de análisis}

18 Esta parte de los análisis se fundamenta en el estudio de los 3453 operadores que han rellenado una ficha Evrest en el año 2009. En primer lugar, los factores de riesgo TME son acotados por la pregunta "Presenta su puesto de trabajo dificultades particulares en los aspectos siguientes : postura / gestos repetitivos / esfuerzos, cargas pesadas", con las modalidades de respuesta por cada uno de los tres tipos de exigencia física "Si, completamente", "más bien sí", "más bien no", o "no, en absoluto".

19 Teniendo en cuenta el número limitado de preguntas en la ficha Evrest, no se puede evidentemente proponer una evaluación precisa de estas regulaciones potenciales (o de 
su ausencia). Para a pesar de todo, aproximarse, las variables utilizadas aquí son las siguientes :

Image

10172E900000557400003862DEF2C5BCF085BE00.emf

Debido à la carga de trabajo, sucede:

¿Tratar con apuro una operación que necesita más cuidado?

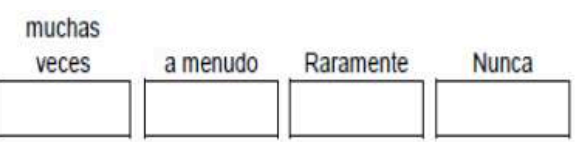

¿Puede usted cotizar las dificultades vinculadas a las cadencias, a los plazos, al cúmulo de tareas, a la variabilidad?

nodifícil

$\begin{array}{llllllllllll}\mathbf{0} & 1 & 2 & 3 & 4 & \mathbf{5} & 6 & 7 & 8 & 9 & \mathbf{1 0}\end{array}$

¿Diria usted que su trabajo presenta las caracteristicas siguientes?

Usted puede elegir la manera de proceder

Usted tiene bastante posibilidades de ayuda mutua, de cooperación

¿Tiene usted la impresión de poder hacer un trabajo de calidad?

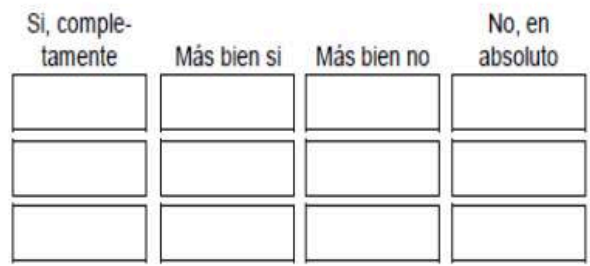

Para hacer un trabajo de buena calidad, dispone usted

- ¿De formación e informaciones suficientes y adecuadas?

- ¿De objetivos y consignas claramente definidas?

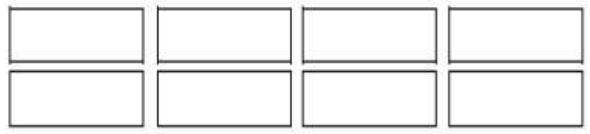

Ciertas regulaciones potenciales interrogan directamente los márgenes de maniobra temporales, mientras que otras se centran más en la capacidad de iniciativa, las cooperaciones posibles, o más generalmente en los medios de asegurar la calidad del trabajo.

Para explorar el aspecto "regulación" en el trabajo de estos operadores estudiando las asociaciones en acción, examinamos a través de una regresión logística los vínculos entre un problema de salud osteo-articular (variable a explicar) y variables explicativas: por una parte los factores de riesgo TME y la edad, y por otra parte las variables de regulación potenciales elegidas. Manteniendo el resto de factores iguales, queremos ver si las variables de regulación son susceptibles de "proteger" contra los problemas osteo-articulares, mirando igualmente los efectos de la edad y las exigencias físicas al ser factores "agravantes". Se presupone aquí un sentido causal que no es verificado por la regresión en sí misma sino por hipótesis resultantes de la literatura ergonómica que evocamos.

Se puede, con los datos de Evrest, tratar las cuestiones de regulación también desde otro punto de vista. Las preguntas sobre la existencia de problemas de salud presentes en la ficha están todas acompañadas de la cuestión: “¿es éso una molestia en el trabajo ?" (respuesta "sí" o "no"). Entonces también es posible estudiar las regulaciones eventuales frente a las molestias en el trabajo en los operadores afectados por un problema de salud. Para eso, se desplaza el modelo anterior : el hecho de estar molesto en el trabajo por un problema de salud osteo-articular es la variable a explicar, y esta vez no nos situamos en la población del conjunto de los operadores si no en la población de los que están afectados por este problema de salud. En el año 2009, 976 operadores presentan problemas de vértebras, 538 de miembros superiores. 


\subsection{Resultados}

Cuando se aplica el modelo que "explica" los problemas de vértebras, se averiguan primero los efectos agravantes de la edad y de las exposiciones físicas. Pero ciertas variables parecen desempeñar un papel de regulación (o protector, según el vocablo normativo en epidemiologia), puesto que van con odds-ratios (OR) inferiores a 1 (figura 1) : los márgenes de tiempo en la acción ("no - más bien no o en absoluto- tratar con apuro una operación que necesita más cuidado"), las posibilidades de organizarse ("tener - más bien o completamente - la elección de la manera de proceder"), y la claridad de los objetivos asignados y de las consignas recibidas (solo en tendencia, pues en el umbral de 0,05 que hemos utilizado aquí la significatividad no es perfecta). "Las posibilidades de cooperación" parecen en cambio tener un efecto más bien agravante.

Por otro lado, cuando se hace el mismo análisis explicando los problemas de miembros superiores, ninguna variable destaca claramente como medio de regulación eficaz (Buchmann, 2013) [?].

Figura 1 : Posibilidades de regulaciones e influencias de las exigencias físicas y de la edad, con respecto a los problemas de vértebras (operadores 2009)

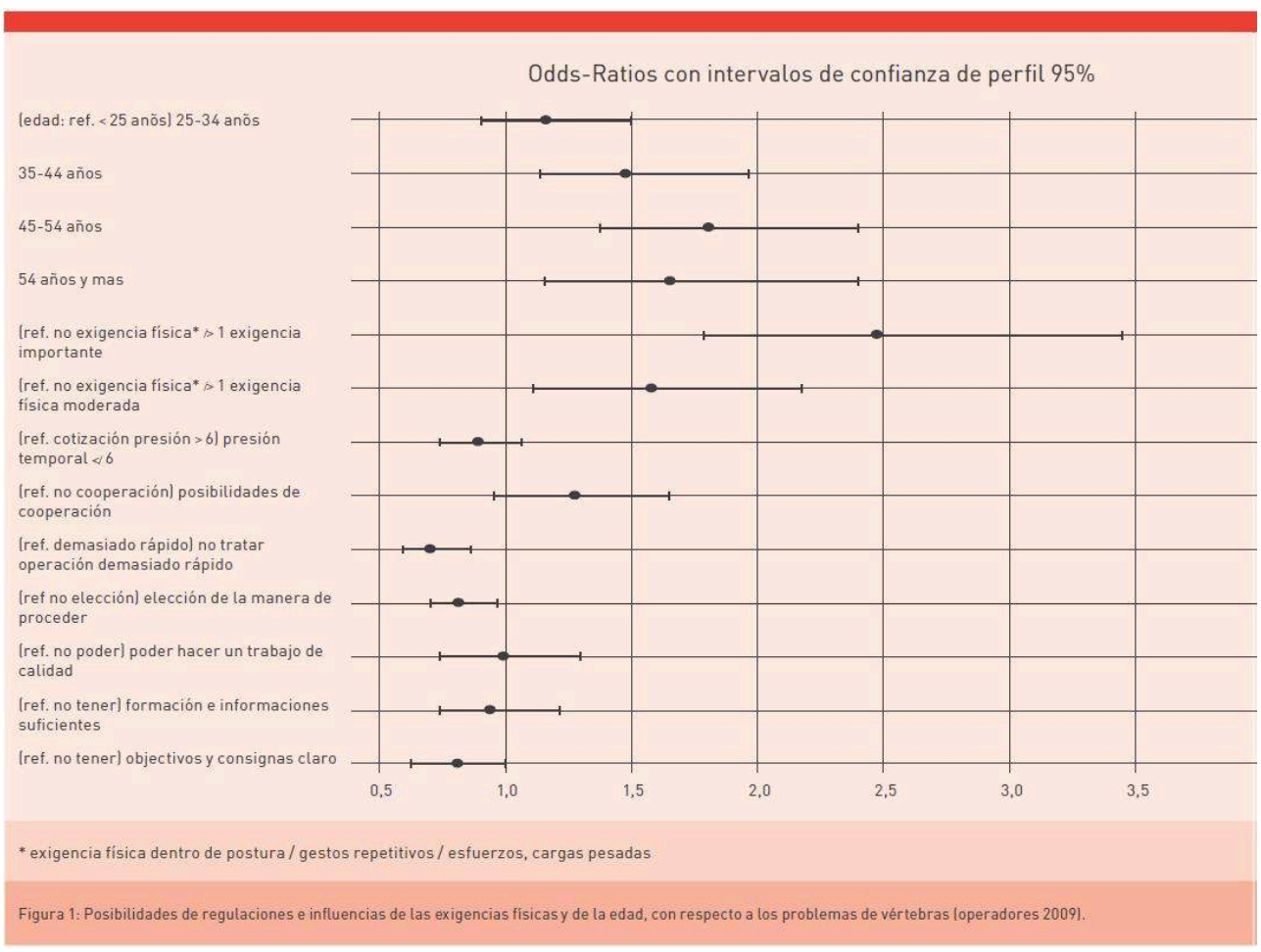

Cuando se considera las eventuales regulaciones posibles con respecto a las molestias en el trabajo (Buchmann, 2013), para los operadores afectados por problema de vértebras, "no tratar con apuro una operación que necesita más cuidado" destaca de nuevo como variable "protectora" ( $O R=0.7$, significativamente diferente de 1 ), es decir, que disminuye la probabilidad de estar molesto en el trabajo por estos problemas de vértebras, independientemente de la edad y de las exigencias físicas que aumentan esta probabilidad. Para los operadores afectados a nivel de los miembros superiores, la impresión de que pueden hacer un trabajo de calidad desempeña este papel con respecto a las molestias en el trabajo $(\mathrm{OR}=0.4)$. 


\subsection{Discusión acerca de este enfoque a través de los mecanismos de "regulación"}

26 A la vista de estos resultados, existen entonces en ciertas situaciones de trabajo de esta empresa posibilidades de regulaciones de las exigencias físicas con respecto a problemas osteo-articulares, que pasan por diversos aspectos de la organización del trabajo. También se dan (a pesar de ser menos numerosas) regulaciones posibles con respecto a las molestias en el trabajo para los que ya están afectados por un problema osteo-articular.

Por otro lado, los miembros superiores y las vértebras no se pueden considerar de manera equivalente con respecto a estos mecanismos de regulación.

El efecto agravante de la imposibilidad de cooperación sobre la probabilidad de tener problemas de vértebras no constituye del todo una sorpresa en este tipo de producción, donde el trabajo en cooperación va a menudo acompañado de exigencias para que cada uno se adapte a los gestos y ritmos de los colegas (Millanvoye \& Colombel, 1996).

En cuanto al papel protector del "trabajo de calidad" con respecto a las molestias debidas a los dolores del miembro superior, se puede avanzar la explicación siguiente : cuando las herramientas, los materiales, y también la organización son concebidos de manera adecuada, se puede a la vez alcanzar objetivos de calidad (sumamente elevados, en la construcción aeronáutica, por razones de coste de los productos, y de seguridad de las aeronaves fabricadas), y adoptar estrategias gestuales que protejan el organismo.

\section{Los mecanismos de "desgaste" : a medio plazo, ¿qué huellas en la salud del "esfuerzo" repetido o prolongado?}

\subsection{Repasos conceptuales}

Lo hemos dicho, cuando las posibilidades de regulación no son lo bastante protectoras, particularmente porque la organización y los medios de trabajo no dejan bastantes márgenes de maniobra individuales y colectivos para desplegarlas, hay desgaste de los operadores en el trabajo (Krause, Rugulies, Ragland \& Syme, 2004). Considerar la acumulación de los "esfuerzos" a través del tiempo es una manera de aprehender los fenómenos de desgaste eventualmente activos en la empresa.

31 Las elecciones hechas aquí para definir el "esfuerzo" se apoyan en la literatura sobre los factores de riesgo TME (Harkness, Macfarlane, Nahit, Silman, \& McBeth, 2003; Ijzelenberg, Molenaar, \& Burdorf, 2004), y en particular sobre el modelo de los TME de Buchmann y Landry (2010), que identifica varios factores de riesgos TME : factores biomecánicos, factores psicosociales, y factores mecánicos.

En esta investigación, encontramos útil de distinguir las nociones de "exigencia" y de "esfuerzo". Apoyándonos en pistas propuestas por Molinié (2003) o Falzon (2004) [8], dentro de una visión del operador activo en su ambiente, aprehendemos el "esfuerzo" como una combinación de las exigencias de la situación de trabajo y de las maneras de percibirlas, vivirlas o regularlas por el operador o el colectivo. 


\subsection{Método de análisis} riesgo TME" en dos casos : factores.

Según la lógica de lo que antecede, se puede incorporar la falta de posibilidades de regulaciones en la propia definición del "esfuerzo".

Para eso, una variable de "esfuerzo en relación a los factores de riesgo TME" fue construida, a partir de varias cuestiones de Evrest: tres cuestiones sobre de la carga física del puesto de trabajo (cuáles son las dificultades relacionadas con las posturas ; con los gestos repetitivos ; y con los esfuerzos y cargas pesadas), también la cuestión de la posibilidad de elegir la manera de proceder, y una puntuación - de 0 a 10 - de las dificultades debidas a la presión temporal, a partir de la escala analógica utilizada en la ficha. Se considera un operador sometido a "esfuerzos en relación a los factores de

- si contestó "sí, importantes" a una de las tres cuestiones sobre la carga física de trabajo, en este caso esta respuesta basta para considerar que existe un factor de riesgo ;

- si contestó "sí, moderadas" a una de estas cuestiones mientras puntuó como mínimo con un 7 sobre 10 las dificultades relacionadas a la presión temporal, y declaró no tener elección en la manera de proceder (respuestas "más bien no" o "no, en absoluto") ; se trata entonces de considerar que la carga física no es en sí misma un factor seguro de riesgo, sino que sus consecuencias son agravadas por la obligación de apurarse y la falta de margen de maniobra.

En coherencia con el modelo precitado, la variable de "esfuerzo" construida se apoya en factores biomecánicos, traducidos aquí por las tres preguntas sobre la carga física del puesto de trabajo; y sobre factores psicosociales, con la cuestión de la elección de la manera de proceder y con la escala analógica de puntuación de las dificultades debidas a la presión temporal. En cambio, ningún factor mecánico (presión, choques, vibraciones) figura en el cuestionario de Evrest, pero aquí no son excesivamente necesarios, pues los análisis de la actividad realizados in situ o los documentos de prevención de riesgos revelan únicamente exposiciones muy marginales a estos

Para poner en relación la salud osteo-articular actual y el "esfuerzo" a lo largo del tiempo, creamos una variable de "secuencia de esfuerzo" de los individuos, y consideramos los lazos entre esta trayectoria y su salud actual.

Con este objetivo, hemos limitado aquí el estudio a la sub-población de los operadores vistos como mínimo tres veces, durante un lapso de tiempo lo más amplio posible, para poner en relación "secuencias de esfuerzo" y su relación con la salud. Se trata de ver lo que ocurre a un individuo, según si es "con esfuerzo" o "sin esfuerzo" en tres fechas sucesivas. La fecha t3 corresponde a una ficha Evrest rellenada en 2008 o 2009 (por los operadores que fueron vistos ambos años, se toma en cuenta la ficha más reciente). Simétricamente, la fecha t1 corresponde al año 2002 o 2003, y esta vez es la ficha más antigua la que se conserva para los operadores vistos ambos años, la idea es de poner una distancia máxima entre $t 1$ y $t 3$. La fecha $t 2$ es mediana: se trata de un Evrest rellenado en 2005 o 2006, guardando la ficha más reciente por los individuos vistos ambos años. Una variable de "secuencia de esfuerzo" fue construida para esta población de 1117 operadores, con 8 combinaciones posibles, yendo desde los "con esfuerzo" en las tres fechas a los "sin esfuerzo" en las tres fechas.

Por una parte, cruzamos estas "secuencias de esfuerzo" con la salud de los operadores en la fecha t3. Por otra parte, insertamos estas "secuencias de esfuerzo" como variables 
explicativas ajustadas a la edad de regresiones logísticas explicativas de los problemas de vértebras y de miembros superiores.

Una segunda manera de tratar los mecanismos de desgaste es examinar más generalmente la "acumulación de esfuerzo" de los operadores en el conjunto de los años observados.

La población considerada aquí es más vasta que la precedente, pues engloba todos los operadores presentes en 2008-2009, o sea 6141 individuos que rellenaron una ficha Evrest como mínimo uno de estos dos años (conservando aquí también los datos más recientes para los que hubieran sido vistos ambos años). La idea era de definir para esta población un indicador de "acumulación de esfuerzo" a lo largo del tiempo. Por eso, no era aceptable contar únicamente cuántas situaciones de "esfuerzo" se constataban a lo largo del tiempo para esta población, pues este número depende de cuántas veces los operadores rellenaron una ficha Evrest desde 2002, número de veces variable de una persona a otra en función del tiempo que lleve en la empresa, la fecha de entrada de su entidad en el dispositivo Evrest, la periodicidad y el calendario de las visitas médicas que pasó, etc. Para superar esta dificultad, el método adoptado es pasar todos los años de 2002 a 2009, incrementando el indicador en 1 cada vez que hay "esfuerzo", y restando 1 al indicador cada vez que no hay "esfuerzo" (figura 2). El valor de este indicador varía entonces de -8 (para los que hubieran sido vistos 8 veces sin jamás estar "con esfuerzo") a +8 (para los que hubieran sido vistos los 8 años estando "con esfuerzo" cada vez). El valor 0 , por ejemplo, corresponde a los operadores vistos un número par de años, y que estuvieron las mismas veces "con esfuerzo" que "sin esfuerzo".

Figura 2 : Ejemplos de cálculo del indicador de "acumulación de esfuerzo".

\begin{tabular}{|c|c|c|c|c|c|c|c|c|}
\hline 2003 & 2003 & 2004 & 2005 & 2006 & 2007 & 2008 & 2009 & \\
\hline cE & $\mathrm{cE}$ & $c E$ & $c E$ & $c E$ & $c E$ & $c E$ & $c E$ & $\rightarrow$ acumulación $=8$ \\
\hline$\underline{s E}$ & $\underline{S E}$ & $\underline{S E}$ & $\underline{S E}$ & $S E$ & $\underline{s E}$ & $\underline{s E}$ & $\underline{s E}$ & $\rightarrow$ acumulación $=-8$ \\
\hline$c E$ & $\underline{S E}$ & $X$ & $c E$ & X & $\mathrm{cE}$ & $\underline{S E}$ & $\underline{S E}$ & $\rightarrow$ acumulación $=0$ \\
\hline$X$ & $X$ & $\underline{s E}$ & $S E$ & $\underline{s E}$ & X & $S E$ & $S E$ & $\rightarrow$ acumulación $=-5$ \\
\hline$X$ & $X$ & X & $X$ & $X$ & $X$ & $\underline{s E}$ & $\underline{s E}$ & $\rightarrow$ acumulación $=-2$ \\
\hline$c E$ & $X$ & $c E$ & $\underline{s E}$ & $\underline{S E}$ & $\underline{s E}$ & $\underline{s E}$ & $X$ & $\rightarrow$ acumulación $=-2$ \\
\hline$c E$ & $S E$ & $c E$ & $c E$ & $c E$ & $S E$ & $c E$ & $c E$ & $\rightarrow$ acumulación $=4$ \\
\hline$\frac{\delta}{\mathrm{d}}$ & $\begin{array}{l}c E \\
\underline{\underline{s E}} \\
X\end{array}$ & \multicolumn{6}{|c|}{ "sin esfuerzo" a la fecha considerada } & \\
\hline & & side & uld & dica & de " & & & \\
\hline
\end{tabular}


41 Dos regresiones logísticas han sido realizadas, una explicando los problemas de vértebras, la otra los problemas de miembros superiores en los operadores interrogados con Evrest en 2008-2009. La "acumulación de esfuerzo", en cinco clases, figuraba como variable explicativa, estando controlada la edad de los operadores (en cinco clases igualmente).

\subsection{Resultados}

42 La población “con esfuerzo » representa más o menos un operador sobre dos, con variaciones ligeras e irregulares de un año al otro; la prevalencia de los dolores de miembros superiores o de vértebras es también aproximadamente estable (del orden del $20 \%$ y $30 \%$ de los operadores respectivamente). La "secuencia de esfuerzo" definida fue cruzada con la salud en $\mathrm{t} 3$, tratándose de los problemas de vértebras por una parte, y de los problemas de miembros superiores por otra (figura 3).

43 Se constata que los operadores con la prevalencia de problemas de vértebras más importante (con un OR significativamente diferente de 1) son los que estuvieron "con esfuerzo" como mínimo dos de tres veces, una de ellas la última. De manera previsible, los "tres veces sin esfuerzo" son los que presentan en proporción la menor cantidad de problemas de vértebras.

En relación a los miembros superiores, la prevalencia más elevada atañe, como se podía prever, a los operadores tres veces "con esfuerzo", y la menos elevada, de nuevo los "tres veces sin esfuerzo". Los "sin esfuerzo" al principio y siempre "con esfuerzo" después, presentan también una prevalencia elevada, como era el caso para las vértebras. Los operadores "con esfuerzo" únicamente en la última fecha (secuencia sE$\mathrm{sE}-\mathrm{cE}$ ) presentan también una prevalencia notable de problemas de miembros superiores. 
Figura 3 : Problemas de vértebras y de miembros superiores en $\mathrm{t} 3$, para cada "secuencia de esfuerzo".

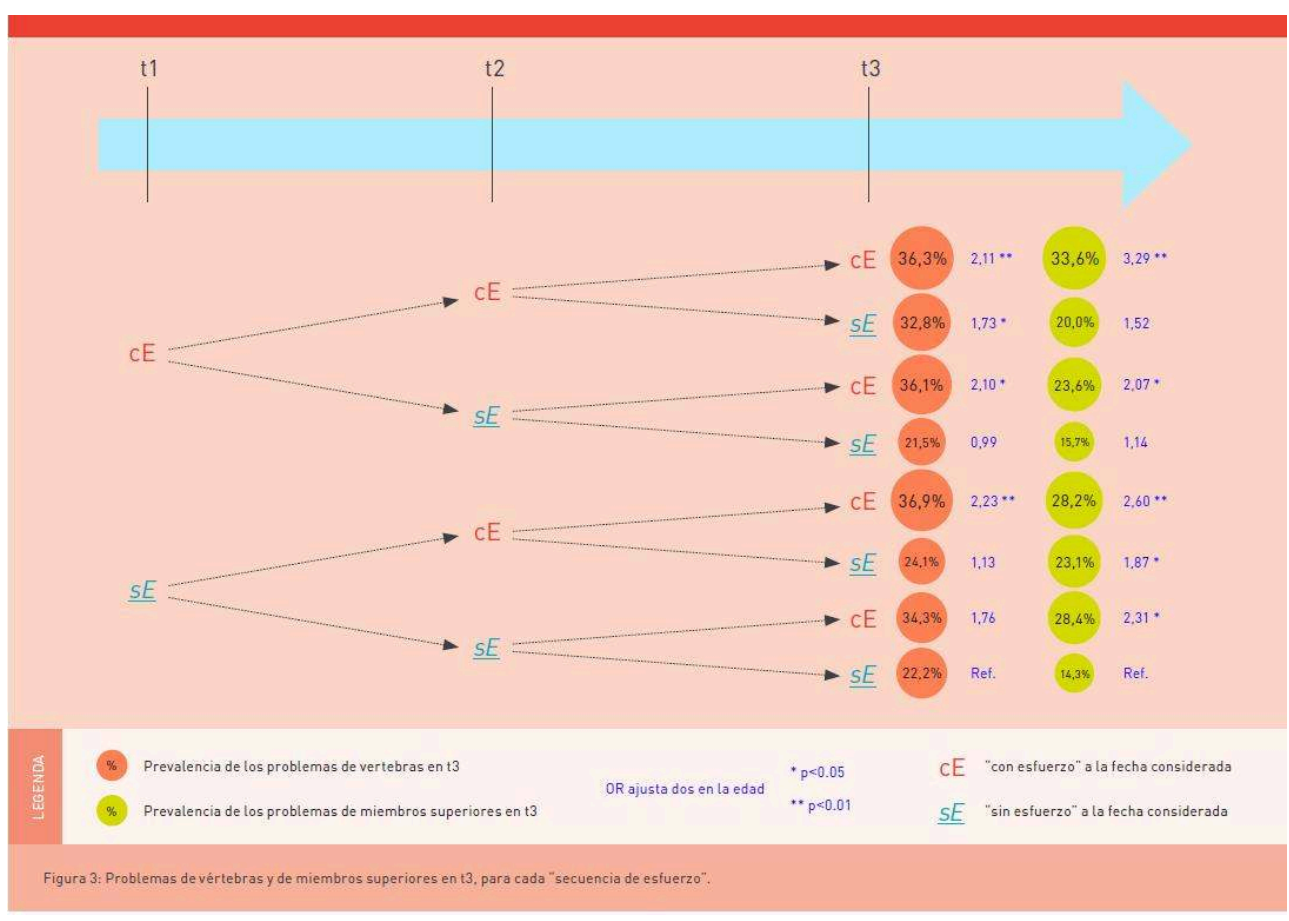

Lectura del gráfico : de los operadores "con esfuerzo" en las tres fechas (secuencia cE-cE-cE), $36.3 \%$ presentan problemas de vertebras en $\mathrm{t} 3$ (OR de 2.1 con respecto a la referencia sE-sE-sE,

independientemente de la edad). De estos mismos operadores $33.6 \%$ presentan problemas de miembros superiores en $\mathrm{t} 3$ (diferencia aún más pronunciada con la referencia sE-sE-sE, pues el OR vale 3.29).

La figura 4 presenta los resultados de las regresiones logísticas explicativas de los problemas de vértebras y de los problemas de miembros superiores por los valores de la "acumulación de esfuerzo", ajustados a la edad. Los valores de "acumulación" por debajo de -1 aparecen como "protectores", específicamente para los problemas de vértebras (de manera significativa, pues el intervalo de confianza no comprende el valor 1). Se observa entonces que cuanto más bajo es el indicador de "acumulación", más reducida es la probabilidad de ocurrencia de problemas osteo-articulares, y al contrario cuanto más alta es la "acumulación", más aumenta esta probabilidad. Entonces cuanto más se acumula "esfuerzo" a lo largo del tiempo, más importante es la probabilidad de tener un problema osteo-articular. 
Figura 4 : Odds-ratios (e intervalos de confianza a $95 \%$ ) para cinco clases de "acumulación de esfuerzo", en dos regresiones logísticas explicativas por una parte de los problemas de vértebras y por otra parte de los problemas de miembros superiores, ajustados a la edad (en 5 clases), entre los operadores vistos en 2008-2009.

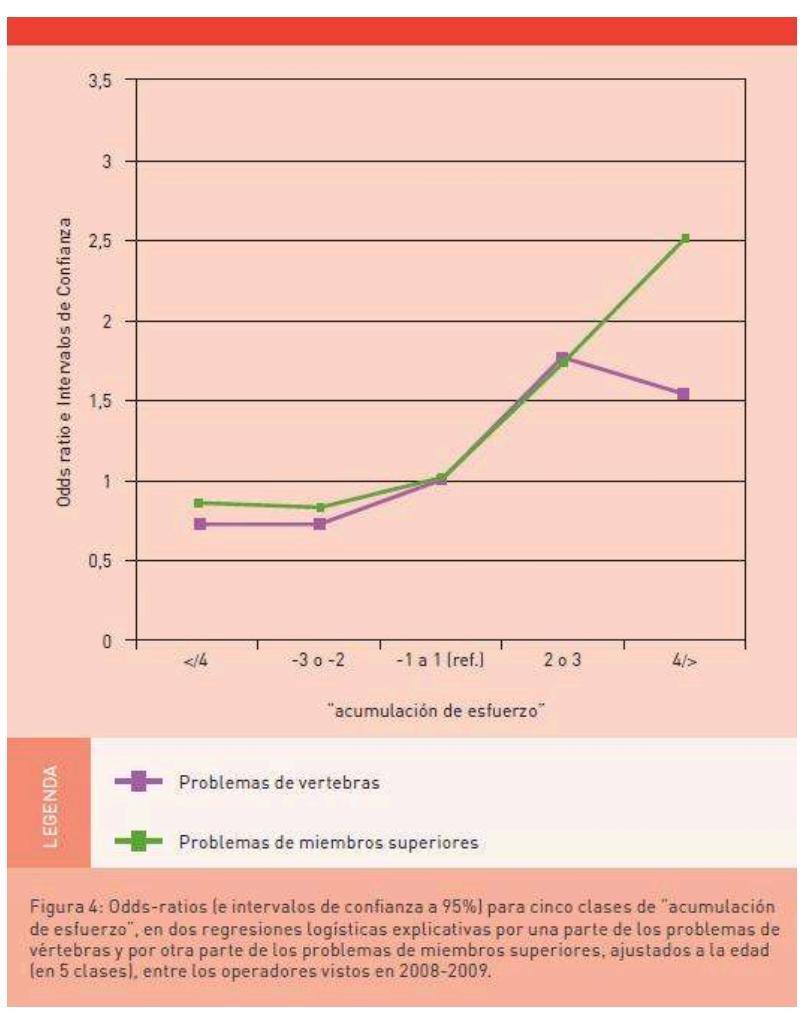

\subsection{Discusión acerca de este enfoque por los mecanismos de "desgaste"}

La constatación de la ausencia de una tendencia clara a lo largo del tiempo de la prevalencia de "esfuerzo" se basa en comparaciones de la población estudiada en su conjunto, en diferentes fechas, sin mostrar si son las mismas personas que están expuestas o no, enfermas o no, de una fecha a otra. Ahora bien, cada vez que estudios longitudinales han sido realizados a partir de Evrest en esta empresa, constatamos que de una fecha $\mathrm{t} 1$ a otra $\mathrm{t} 2$ había a la vez bastantes individuos que tenían menos problemas de condiciones de trabajo, o de salud, y bastantes para los que la situación había empeorado en uno $u$ otro aspecto, independientemente de que la evolución general vaya en un sentido $u$ otro, o incluso cuando no hay globalmente ninguna evolución entre las dos fechas. Esto confirma el interés de considerar también las relaciones trabajo/salud en su evolución a través del tiempo, integrando las evoluciones individuales.

La comparación de los porcentajes de individuos atañidos por problemas de vértebras por una parte, de miembros superiores por otra parte, en las poblaciones "con esfuerzo" y "sin esfuerzo" nos permitió constatar (Buchmann, 2013) op.cit.) que el hecho de estar actualmente "con esfuerzo" tiene relación con los problemas de salud, y que haberlo estado en el pasado, en cualquier periodo, también está relacionado con la salud actual. En cambio, no hay una tendencia clara que aparezca según la distancia entre el "esfuerzo" pasado y la salud actual, es decir que la relación entre la salud de 
hoy y el "esfuerzo" no es menor dependiendo de si este "esfuerzo" está más o menos lejos en el tiempo. Pero la relación entre trastorno de salud y "esfuerzo" pasado en cualquier fecha, si se analiza por simples tablas cruzadas, puede proceder de otra relación estadística subyacente : el hecho de estar "con esfuerzo" a una fecha está correlacionado evidentemente con el hecho de estar "con esfuerzo" a otra fecha (un operador "con esfuerzo" a un momento dado tiene más probabilidad de estarlo todavía los años siguientes y de haberlo estado los años pasados que un operador "sin esfuerzo" en el mismo momento). La relación aparente entre un "esfuerzo" pasado y la salud actual podría entonces ser solo el "reflejo matemático" de la relación entre el "esfuerzo" actual y la salud actual. Para desembrollar esto, era necesario encontrar métodos para investigar más en detalle las relaciones en el tiempo entre "esfuerzo" y salud, interesándonos especialmente por lo que llamamos la "secuencia de esfuerzo" de los individuos, y considerando las relaciones entre esta secuencia y su salud actual.

Las constataciones realizadas cruzando la "secuencia de esfuerzo" y los problemas de salud permiten barajar la hipótesis de que la repetición del "esfuerzo" en el tiempo es un factor de riesgo muy importante. Es lo que confirma el fuerte gradiente observado al cruzar la "acumulación de esfuerzo" y la salud, lo que desde luego remite a una relación "dosis/efecto" : la repetición del "esfuerzo" indica una exposición de larga duración. Otra interpretación posible de este resultado (que puede ser complementaria a ésta) sería que la repetición del estado "con esfuerzo" podría reflejar en parte la intensidad del "esfuerzo" : si un operador se encuentra en una situación de "esfuerzo" fuerte y estable, se reafirmará de un año a otro en sus respuestas al cuestionario, aunque si este "esfuerzo" es estable pero más moderado, las respuestas del operador a Evrest podrían variar más de una vez a otra, como si reflejaran algo de duda al momento de contestar.

\section{Los mecanismos de "selección" : ¿conducen los dolores articulares a reasignaciones, protegiendo del "esfuerzo" ?}

\subsection{Repasos conceptuales}

Esta parte del artículo relata los procesos estadísticos producidos para examinar los mecanismos de selección en acción en esta empresa, relacionados con los factores de riesgo TME. Con este objetivo, tomamos la salud como factor potencial de reducción del "esfuerzo", la idea es mostrar cómo la salud pasada puede, debido a un mecanismo relativo al "healthy worker effect", mostrarse predictiva de exigencias de trabajo actuales, o sobre todo de su ausencia, después de una "salida de exigencia" de los trabajadores que presentan trastornos de salud.

\subsection{Método de análisis}

Observamos las relaciones entre los dolores articulares en los operadores "con esfuerzo" en una fecha y su "esfuerzo" en una fecha ulterior, en dos sub-poblaciones : por una parte los 1249 operadores vistos en 2002 o 2003 y vistos también en 2008 o 2009 (para trabajar con un lapso de tiempo bastante amplio), y por otra parte los 2471 operadores vistos en 2005 o 2006 y vistos también en 2008 o 2009 (para trabajar con una población más grande). 
Hemos realizado el mismo análisis "endureciendo" el criterio de salud tomado en cuenta: hemos comparado las proporciones de operadores sustraídos al "esfuerzo" entre dos fechas según estuvieran o no sujetos a dolores y limitaciones a nivel de las vértebras y de los miembros superiores.

Después hemos querido averiguar si los mecanismos de selección serían aparentes volviendo a los ítems básicos que sirvieron para construir la variable de "esfuerzo". Entonces hemos pretendido ver si la salud en una fecha era predictiva de la evolución de las variables de carga física en el puesto de trabajo que componen nuestra variable de "esfuerzo", siendo dichas variables las dificultades con respecto a las posturas, los gestos repetitivos, y los esfuerzos o cargas pesadas.

\subsection{Resultados}

La figura 5 presenta el porcentaje de operadores de cada "secuencia de esfuerzo" (variable definida más arriba), entre los operadores "con esfuerzo" en la primera fecha, según presentaban o no un problema de salud en esta misma fecha.

Figura 5 : "secuencia de esfuerzo" por venir según la presencia o la ausencia de problemas de vértebras y de miembros superiores en $\mathrm{t} 1$, entre los operadores "con esfuerzo" en $\mathrm{t} 1$ (la tabla contiene porcentajes columnas : la suma de las columnas vale 100).

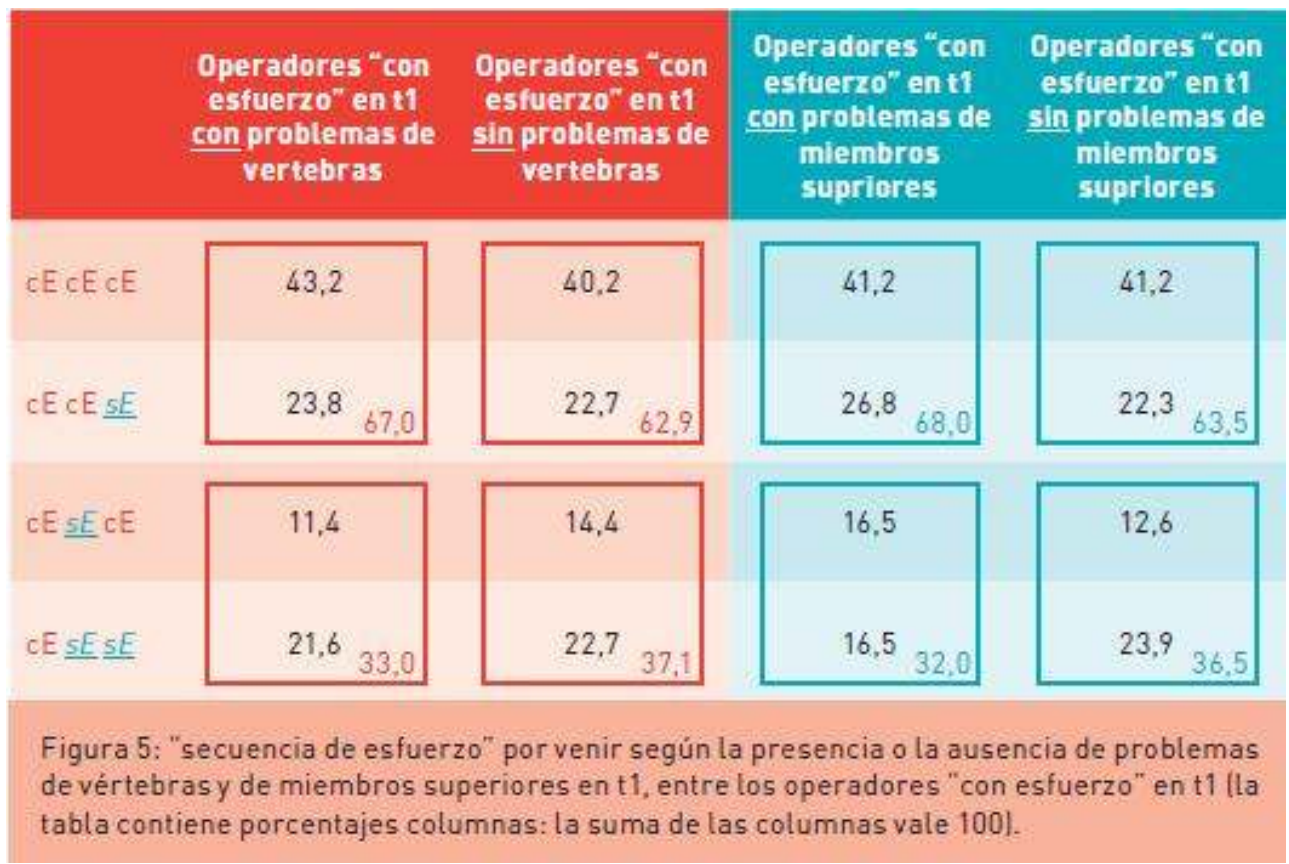

Lectura de la tabla : $43.2 \%$ de los operadores "con esfuerzo" en t 1 y que tenían problemas de vértebras en esta fecha estuvieron también "con esfuerzo" después, en t2 y en t3. Si nos interesamos únicamente en el "esfuerzo" en t1 et en t2, se ve que $67.0 \%$ de los operadores "con esfuerzo" en t1 y que tenían problemas de vértebras en esta fecha estaban también "con esfuerzo" en t2.

Se observan diferencias muy poco importantes en las reparticiones de las "secuencias de esfuerzo" entre los "con esfuerzo" con problemas de vértebras en t1 (columna 1) y los “con esfuerzo » sin problemas de vértebras en $\mathrm{t} 1$ (columna 2). Sin embargo, si se oponen los "con esfuerzo" en $\mathrm{t} 1 \mathrm{y} \mathrm{t} 2 \mathrm{a}$ los "con esfuerzo" en $\mathrm{t} 1$ pero que ya no lo están en $\mathrm{t} 2$ (o sea que oponemos las dos primeras líneas a las dos siguientes), se constata que hay un poco más de operadores "con esfuerzo" en las dos fechas entre los que tienen 
problemas de salud en $t 1, y$ que hay entonces entre ellos menos operadores apartados del "esfuerzo" entre $\mathrm{t} 1 \mathrm{y} \mathrm{t}$ 2, ya se trate de problemas de vértebras o de miembros superiores. Por otro lado, hay muchos menos operadores sustraídos del "esfuerzo" desde $\mathrm{t} 2 \mathrm{y}$ en $\mathrm{t} 3$ entre los "con esfuerzo" en $\mathrm{t} 1$ que tienen problemas de miembros superiores (15.5\%) que entre los que no tienen este tipo de problemas de salud (23.9\%). En contra de lo que se podía esperar aquí, estos resultados no muestran una selección que aparte más del "esfuerzo" los operadores que tienen un problema de salud en $\mathrm{t} 1$; reflejan más bien un ligero fenómeno inverso.

Estrechando el análisis sobre comparaciones únicamente entre dos fechas, por una parte los 1249 operadores vistos en 2002-2003 y en 2008-2009, y por otra parte los 2471 operadores vistos en 2005-2006 y en 2008-2009, se constata en los dos casos que los operadores sustraídos del "esfuerzo" entre las dos fechas no son especialmente más numerosos en proporción a los que tienen un problema de salud al principio (ya sea de miembros superiores o de vértebras), no obstante no se encuentra esta vez una relación inversa bien establecida.

Teniendo en cuenta no las molestias en el trabajo si no las limitaciones ocasionadas por los problemas de salud considerados, observamos aquí un fenómeno de selección entre las dos fechas en relación a las vértebras (aunque no estadísticamente significativo), sobre las dos sub-poblaciones consideradas : $49.0 \%$ de los operadores con limitaciones relacionadas con las vértebras en 2002-2003 estaban sustraídos del "esfuerzo" en 2008-2009, aunque solo estaban $43.2 \%$ entre los que no tenían tales limitaciones (respectivamente $40.7 \%$ y $36.2 \%$ si restringimos la distancia entre las dos fechas : por la sub-población de los operadores vistos a la vez en 2005-2006 y en 2008-2009).

En cuanto a saber si la salud en una fecha dada es predictiva de la evolución de las variables de carga física en el puesto de trabajo componiendo nuestra variable de "esfuerzo", un resultado en este sentido se encuentra efectivamente en las dos subpoblaciones de operadores (diferentes según las fechas utilizadas). Tener un problema de miembros superiores, entre los expuestos a los esfuerzos o a cargas pesadas en la primera fecha, está relacionado con el hecho de ya no estar expuestos a ello en la secunda fecha : $80.9 \%$ de los operadores con un problema de miembros superiores en 2002-2003 fueron sustraídos de esta exigencia física entre las dos fechas, aunque solo estaban $69.7 \%$ entre los que no presentaban problemas a este nivel, con $\mathrm{p}=0.15$ por el test del $\chi 2$ (respectivamente $65.6 \%$ y $58.6 \%$ por la sub-población de los operadores vistos a la vez en 2005-2006 y en 2008-2009, $p=0.16$ ). Sin embargo, no se observa tal cosa en cualquier nivel para las dos otras exigencias físicas, ni por otro lado por los problemas de vértebras, cualquiera que sea la exigencia estudiada.

\subsection{Discusión acerca de este enfoque por los mecanismos de "selección"}

58 En general entonces, no se observa una selección respecto al "esfuerzo" tal y como lo definimos, excepto eventualmente en relación a las limitaciones relativas a las vértebras, y se constata que un fenómeno de selección parece producirse con respecto a los esfuerzos y a las cargas pesadas relacionados con los problemas de miembros superiores.

59 Se puede proseguir aquí la hipótesis, ya propuesta a propósito de nuestros análisis del "desgaste", que un operador "con esfuerzo" en una fecha y que ya no lo está después 
(en prolongación, sin duda, de un anterior vaivén entre "esfuerzo" y "no esfuerzo") lo está menos intensamente que un operador que parece "con esfuerzo" en ambas fechas (y quizás "con esfuerzo" continuo anteriormente), siendo éste por lo tanto más susceptible de presentar dolores articulares. Explorar esto depende más bien de análisis monográficos, llevados durante esta investigación pero que no tienen la vocación de ser presentados en el presente artículo.

\section{Aportes y límites de un análisis estadístico de los dolores articulares en la empresa}

60 En las empresas - al menos en Francia - el único recuento usual de los problemas articulares es el número de casos de enfermedad profesionales (EP) reconocidos por la Sécurité Sociale, y que conciernen las afecciones de los miembros superiores o de la espalda. Dijimos en introducción, y subrayamos aquí, que su declaración y su reconocimiento relevan de un procedimiento delicado, al término de cual solo una pequeña parte de las afecciones debidas al trabajo es efectivamente evaluada. En la empresa estudiada aquí, es manifiesto : como indicado, la prevalencia de los dolores de vértebras entre los operadores de esta empresa se aproxima a los $30 \%$, y a los $20 \%$ para los dolores de miembros superiores, en relación una población de aproximadamente 10000 operadores del conjunto del grupo - aunque el número de TME reconocidos, en una fecha dada en esta empresa es inferior a 200, en torno a un décimo de la población está afectada por dolores.

61 El orden de magnitud de los TME reconocidos, y de los dolores articulares, no son equivalentes y se puede admitir que éstos últimos, además del hecho de que su gran número autoriza análisis cuantitativos detallados, constituyen por sí mismos un problema legítimo de salud laboral. Es el punto de vista que adoptamos en esta investigación, y la importancia de las relaciones entre características del trabajo y la sobrevenida de estos trastornos nos incita en ello : para seguir en los resultados de la figura 2, se puede aceptar la idea de que una exposición prolongada al "esfuerzo", tal y como lo definimos (como mínimo una exigencia física de nivel importante, o al menos una de nivel más moderado pero combinada con exigencias organizacionales), se acompaña de probabilidades incrementadas de desarrollar trastornos vertebrales (OR aproximadamente de 2, tras ajuste en la edad) o de miembros superiores (OR que puede llegar a 3.7) - a pesar de que todo este análisis se ubica en la población de los operadores, es decir del personal de taller, entre los cuales ninguno está realmente exento de solicitaciones físicas.

La tercera parte de nuestro análisis, que se refiere a la "selección", acrecienta las preocupaciones que acabamos de evocar. Demuestra en efecto que dentro de este medio profesional con oficios precisamente definidos, no hay mucha movilidad protectora para los operadores, numerosos, afectados por dolores articulares; en todo caso nada muy específico: mutaciones de situaciones "con esfuerzo" a "sin esfuerzo" tienen lugar, sobre todo para operadores en segunda parte de carrera (como ya ha podido ser constatado), pero solamente con una baja frecuencia en forma de reasignación determinada por razones de salud. Nuestras observaciones más monográficas en el terreno lo han confirmado : en un establecimiento de varios centenares de operadores, repasando con el medico del trabajo los historiales médicos de operadores que habrían 
sido mutados de puesto de trabajo en razón de sus dolores articulares, pudimos reunir nueve casos en total, en un periodo de varios años.

63 A fin de cuentas, las reasignaciones constituyen, como lo dijimos, solo un último recurso, pues son portadoras de un incremento de riesgos para los operadores todavía con buena salud a través de una sobrexposición. La disminución del número de operadores "con esfuerzo" sigue siendo entonces un objetivo esencial, sobre todo en un contexto de envejecimiento de la población. Recordemos aquí que esta categoría de los "con esfuerzo" es definida por nosotros, tras considerar los conocimientos disponibles sobre todo en ergonomía, a partir de una combinación entre exigencias físicas (posturas, repeticiones, esfuerzos y cargas pesadas) y la restricción de los márgenes de maniobra en la actividad de trabajo (presión temporal, falta de margen de maniobra). Tratándose de esfuerzos físicos, ciertas posibilidades de mejora ya han sido ampliamente utilizadas en esta empresa; nuevos progresos son ciertamente posibles, pero en el sector de la construcción aeronáutica una implicación importante del cuerpo en el trabajo es difícil de evitar. La primera parte de nuestro análisis estadístico nos indica que una acción sobre los aspectos organizacionales, una cierta disminución de las exigencias de tiempo, un incremento de las posibilidades de elegir la propia manera de proceder, una apreciación más fiable de los objetivos de trabajo, constituyen medios de prevención que requieren atención : una situación "positiva" en estos tres aspectos a la vez (recordemos que en este caso los OR se multiplican), va acompañada de un OR global de 0.44 para la presencia de dolores de vértebras, es decir, de una división entorno a dos de su prevalencia, siendo fijos la edad y el nivel de exigencias físicas. Desde este punto de vista, un atento examen de las reorganizaciones pendientes en este sector, y específicamente de los métodos relativos al Lean Management (Buchmann, Bellies, \& Volkoff, 2010), es particularmente necesario.

Retomando de nuevo algunas conclusiones que acabamos de presentar, se puede considerar que estos enfoques cuantitativos son bastante concluyentes, ya que permiten a la vez llamar la atención sobre factores de perjuicio para la salud, matizar su análisis (todas las relaciones no son de la misma importancia, ni siempre en el sentido esperado), e inscribir el proceso en una perspectiva a medio y a largo plazo. Sin embargo, el tono prudente de las interpretaciones propuestas refleja nuestra preocupación de no considerar que estas cifras "hablan por sí mismas". Para comprender de manera precisa las condiciones en las cuales las regulaciones son desarrolladas (o los límites contra los cuales tropiezan), apreciar las razones por las cuales un operador pudo dar respuestas que lo llevaran a estar clasificado como "con esfuerzo" (o no) a uno u otro periodo de su recorrido, analizar las reasignaciones cuando son posibles - y sus consecuencias individuales y colectivas, hace falta esforzarse en articular los resultados numéricos con análisis de la actividad, y con entrevistas de dimensión retrospectiva. Este es el objetivo global de esta investigación. 


\section{BIBLIOGRAFÍA}

Aptel, M., \& Vézina, N. (2008). Quels modèles pour comprendre et prévenir les TMS ? Pour une approche holistique et dynamique. In Actes du 2ème congrès francophone sur les TMS (pp. 1-27). Montréal, Canada.

Askenazy, P., Cartron ,D., De Coninck, F., \& Gollac, M. (2006). Organisation et intensité du travail. Toulouse : Octarès Editions.

Aublet-Cuvelier, A., Aptel, M., \& Weber, H. (2006). The dynamic course of musculoskeletal disorders in an assembly line factory. International archives of occupational and environmental health, 79(7), 578-584.

Berg, M., Sanden, A., \& Torell, G. (1988). Persistance of musculoskeletal symptoms: a longitudinal study. Ergonomics, 31, 1281-1285.

Buchmann, W. (2013). Aspects de moyen et long termes dans la genèse et l'évolution des Troubles Musculo-Squelettiques au travail. Une recherche dans l'industrie aéronautique (Tesis doctoral no publicado, Tesis doctoral en Ergonomía). CNAM, Paris.

Buchmann ,W., Bellies, L., \& Volkoff, S. (2010, agosto-septiembre). What possibilities for sustainable prevention of WMSD within lean manufacturing production methods?. Proceedings of the $7^{\text {th }}$ international Scientific Conference on Prevention of Musculoskeletal Disorders, PREMUS, Angers, France.

Buchmann, W., \& Landry A. (2010). Intervenir sur les TMS. Un modèle des Troubles Musculosquelettiques comme objet intermédiaire entre ergonomes et acteurs de l'entreprise?.@Activités, $7(2), 84-103$.

Buchmann, W., Mardon, C., Archambault, C., Volkoff, S. (2009, agosto). Combining occupational health data and ergonomics to take early action on Musculoskeletal Disorders. Proceedings of the $17^{\text {th }}$ congress of the International Ergonomics Association (IEA), Beijing, China.

Buchmann, W., Volkoff, S. \& Archambault, C. (2011). L'approche diachronique des TMS, une paire de lunettes pour l'ergonomie myope ?. In F. Jeffroy \& A. Garrigou (Ed.), L'ergonomie à la croisée des risques. SELF'2011, Congrès International d'Ergonomie (pp. 90-95). Paris, France.

Cole, D.C., Manno, M., Beaton, D., \& Swift, M. (2002). Transitions in self reported musculoskeletal pain and interference with activities among newspaper workers. Journal of Occupational Rehabilitation, 12(3), 163-174.

Diricq, N. (2011). Rapport de la commission des accidents du travail et des maladies professionnelles instituée par l'article L. 176-2 du code de la Sécurité Sociale. Retirado de http://www.securitesociale.fr/IMG/pdf/11_diricq.pdf

Eurogip (n.d.). Les TMS en Europe (2007) : définitions et données statistiques. Retirado de www.eurogip.fr

Falzon, P. (dir.) (2004). Ergonomie. Paris : PUF. (Versión española : Falzon, P. (dir.) (2009) Manual de ergonomía. Madrid : Modus laborandi.)

Fauconnier, D., Pépin, M., \& Douillet, Ph. (2005, diciembre). Approche économique de la problématique des troubles musculo-squelettiques. Des coûts aux risques stratégiques. Colloque DARES ANACT, Paris, France. 
Ha, C., Roquelaure, Y. (2010, février). Troubles musculo-squelettiques d'origine professionnelle en France. Où en est-on aujourd'hui ?. BEH numéro thématique TMS, 35-37.

Harkness, E. F., Macfarlane, G. J., Nahit, E. S., Silman, A. J., McBeth, J. (2003). Mechanical and psychosocial factors predict new onset shoulder pain: a prospective cohort study of newly employed workers. Occupational and Environmental Medicine, 60, 850-857.

Ijzelenberg, W., Molenaar, D., \& Burdorf, A. (2004). Different risk factors for musculoskeletal complaints and musculoskeletal sickness absence. Scandinavian Journal of Work, Environment \& Health, 30, 56-63.

Krause, N., Rugulies, R., Ragland, D., \& Syme, S. L. (2004). Physical workload, ergonomic problems, and incidence of low back injury: a 7.5-year prospective study of San Francisco transit operators. American Journal of Industrial Medicine, 46, 570-585.

Laville, A., \& Volkoff, S. (2004). Vieillissement et travail. In P. Falzon (Dir.), Traité d'ergonomie. Paris: PUF.

Mergler, D. (1999). Combining quantitative and qualitative approaches in occupational health for a better understanding of the impact of work-related disorders. Scandinavian Journal of Work, Environment \& Health, 15(4), 54-60.

Millanvoye, M., \& Colombel, J. (1996). Age et activité des opérateurs dans une entreprise de construction aéronautique. In R. Patesson (s/d), Intervenir par l'ergonomie - Actes du XXXI congrès de la Société d'ergonomie de langue française : vol. 2 (pp. 39-46). Bruxelles, Belgique.

Molinié, A-F. (2003). Interroger les salariés sur leur passé professionnel : le sens des discordances. Revue d'Epidémiologie et de Santé Publique, 51, 589-605.

Molinié, A-F., \& Leroyer, A. (2011). Suivre les évolutions du travail et de la santé : EVREST, un dispositif commun pour les usages diversifiés. PISTES, 13(2), 1-25. Tirado en noviembre de http:// www.pistes.uqam.ca/v13n2/articles/v13n2a2.htm

Plouvier, S., Gourmelen, J., Chastang, J. F., Lanoë, J. L., \& Leclerc, A. (2011). Low back pain around retirement age and physical occupational exposure during working life. BMC Public Health, 11, 268.

Silverstein, B., Viikari-Juntura, E., Fan, Z. J., Bonauto, D. K., Bao, S., Smith, C. (2006). Natural course of nontraumatic rotator cuff tendinitis and shoulder symptoms in a working population. Scandinavian Journal of Work, Environment \& Health, 32(2), 99-108.

Vézina, N. (2001). La pratique de l'ergonomie face aux TMS : ouverture à l'interdisciplinarité. In Comptes rendus du $36^{e ̀ m e}$ congrès de la Société d'ergonomie de langue française et du $32^{\text {ème }}$ congrès de l'Association canadienne d'ergonomie [pp. 44-60 (version française) and pp. 39-54 (English version)]. Montréal, Canada.

Volkoff, S., \& Gaudart, C. (2006). Vieillissement et travail. Actualités et dossier en santé publique, 57, 52-54.

Volkoff, S., \& Molinié A.-F. (2011). L'écheveau des liens santé travail, et le fil de l'âge. In A. Degenne, C. Marry et S. Moulin (Dir.), Les catégories sociales et leurs frontières. (pp. 323-344). Laval, Québec : Presses de l'Université Laval.

Volkoff, S., (2012). Dérives et inerties dans la démographie de la population salariée. In A.-F. Molinié, C. Gaudart, \& V. Pueyo (Dir.), La vie professionnelle : âge, expérience et santé à l'épreuve des conditions de travail (pp. 21-30). Toulouse : Octarès, Coll. “ Travail et Activité humaine ». 


\section{ANEXOS}

Anexo : El cuestionario del dispositivo Evrest del grupo aeronáutico estudiado (versión 2009)

Image 1019D3280000466700002D25BC389F8CAA64BCE1.emf

\section{CONDITIONS DE TRAVAIL}

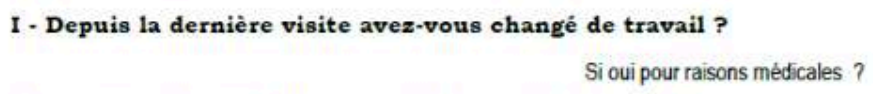

Oui

Oui

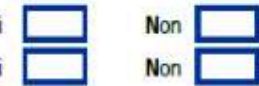

II - Quels sont habituellement vos horaires de travail ? Tps plein

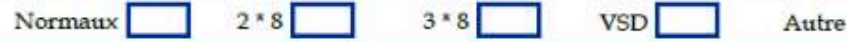

Tps partiel

\section{III - Contraintes de temps :}

- En raison de la charge de travail, vous arrive t'il de

Dépasser vos horaires normaux Sauter ou écourter un repas, ne pas prendre de pause Traiter trop vite une opération qui dernanderait davantage de soin

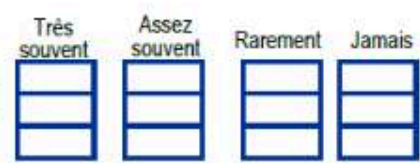

- Pouvez-vous coter les difficultés liées aux cadences, aux délais, au cumul de tâches, à la variabilité

Pas difficile

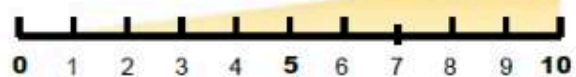

Très difficile faire pour une autre non prévue ?

- Si OUI diriez-vous que cette interruption d 'activité : (1 serule rếponse)

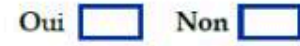

Perturbe votre travail Est sans conséquences pour votre travail Est un aspect positif de votre travail

Image 101D4450000046C00000330CA965F9C5A1FED256.emf

IV-Appréciations sur le travail : Diriez-vous que votre travail présente les caractéristiques suivantes ?

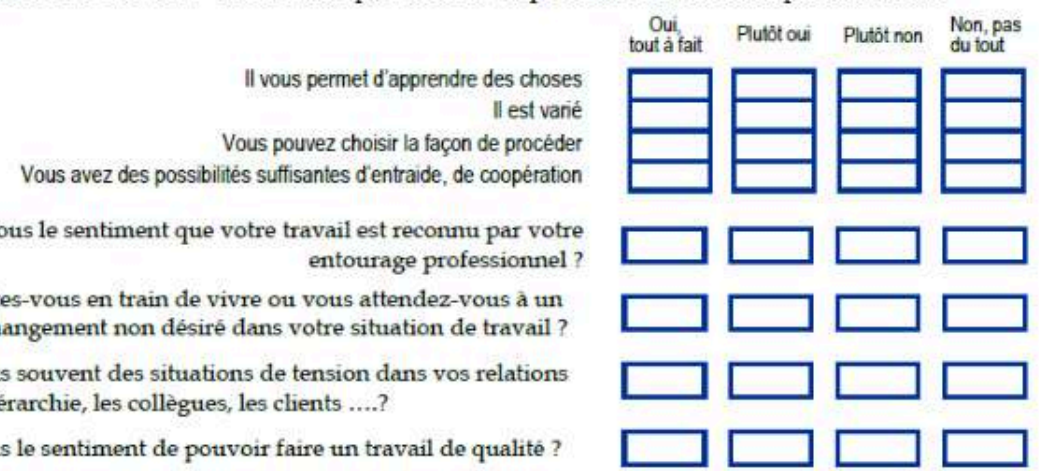
avec la hiérarchie, les collègues, les clients ....?

Avez-vous le sentiment de pouvoir faire un travail de qualité ?

Pour faire un travail de bonne qualite disposez-vous :

De formation et $d$ 'informations suffisantes et adéquates $D$ 'objectifs et de consignes clairement définies

V - Charge physique du poste de travail

Votre poste de travail présente $t$ ' il sur les plans suivants
Posture

Importants déplacements à pied Gestes répètitifs

Efforts, charges lourdes Station debout prolongée

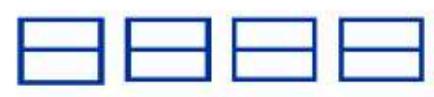


Image 1019BD48000046C000002CE2A0D81C24A2E81BC0.emf VII - Avez- vous des déplacements professionnels :
Oui
Non $\square$

Si oui :

Moins $\mathrm{d}^{\prime} 1$ fois/semaine

1 fois/semaine

Plus de 2 fois/semaine

Avez-vous eu des missions hors CEE depuis la dernière visite?

Oui

Trouvez-vous ces déplacements difficiles ?

Oui $\square$

\section{FORMATION}

I - Depuis la dernière visite avez - vous eu une formation?

Si oui, était-ce une formation votre poste de travail ? $\square$ un futur poste? $\square$

d'intérêt général? en rapport avec

II - Avez-vous eu depuis la dernière visite un rôle de formateur, de tutorat ?

Oui $\square$ Non

\section{MODE DE VIE}

I - Pratiquez-vous de façon occasionnelle ou régulière une activité sportive ? Oui $\square$ Non $\square$

Si oui : $\quad$ Moins $d^{\prime} 1$ fois/semaine $\square 1$ à 2 fois/semaine

$\square$

Plus de 2 fois/semaine $\square$

II - Consommations usuelles dont le Week End

\begin{tabular}{|l|c|c|c|c|}
\hline Tabac en cigarettes/3 & 0 & Moins de 5 & 5 à 15 & Plus de 15 \\
\hline Café en tasse(s) $/ \mathrm{j}$ & 0 & 1 & 2 à 4 & Plus de 4 \\
\hline Alcool en verres $/ \mathrm{j}$ & 0 & Moins de 2 & 2 à 4 & Plus de 4
\end{tabular}$\quad$ Ex fumeur Oui $\quad$ Non $\square$

Image 10249FE0000046C000003FFB8495BD0035BFCD0C.emf

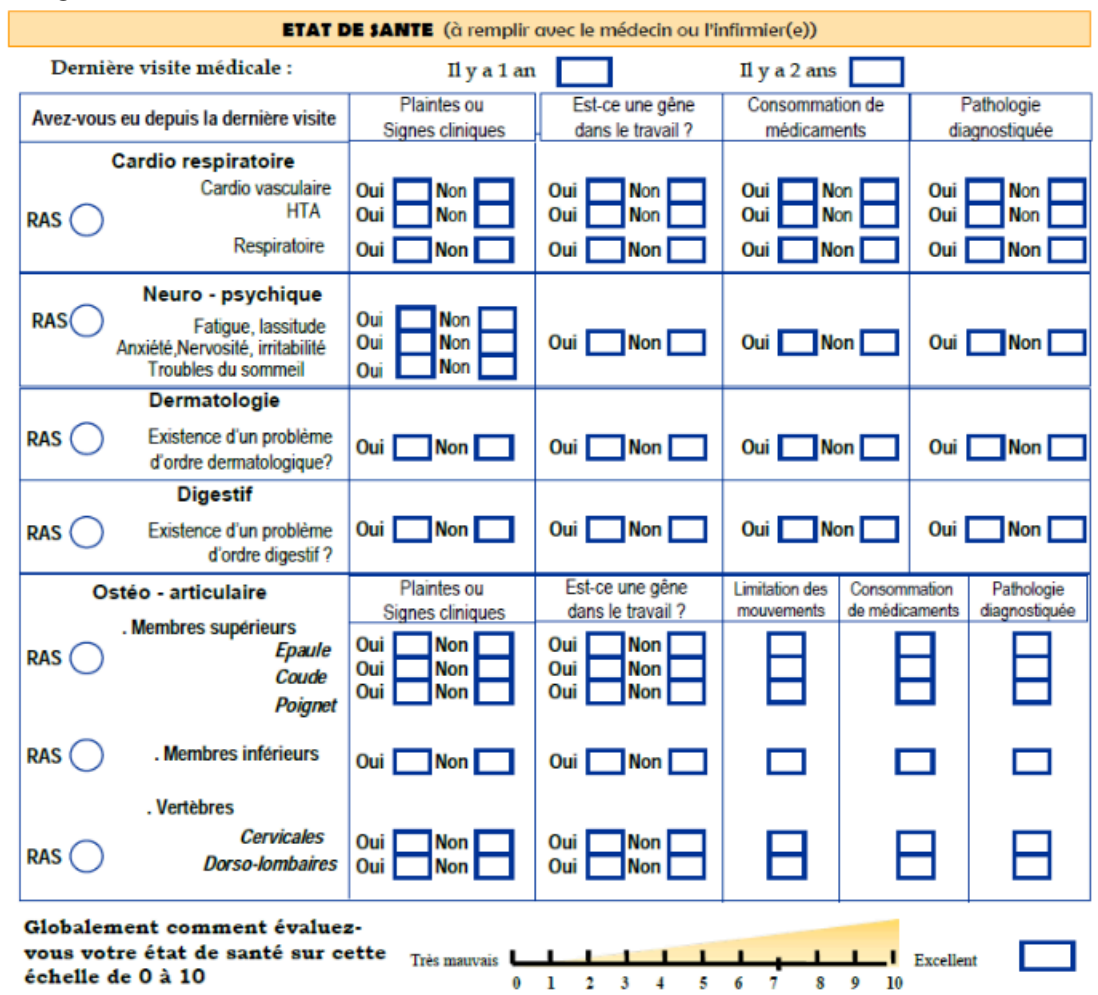

\section{NOTAS}

1. "Caisse nationale de l'assurance maladie" léase Seguridad

2. Evoluciones y relaciones en salud - trabajo

3. Este cuestionario es presentado en su integralidad en anexo (en francés). 
4. Seguidamente, el término "operador" designara los personales de taller, del agente de fabricación al jefe de línea por ejemplo, y en oposición a las profesiones administrativas y recursos humanos.

5. Definiremos entonces este término.

6. Ver nota precedente

7. Para limitar el tamaño de este artículo, ciertos resultados de la investigación no pueden ser presentados íntegramente, aunque es importante evocarlos. En casos similares nos referiremos a la tesis.

8. Los términos "exigencia" y "esfuerzo" utilizados aquí provienen de la traducción en español de Falzon (2004).

\section{RESÚMENES}

Este artículo está basado en la parte cuantitativa de una investigación en ergonomía cuyo objetivo es comprender cómo evoluciones del trabajo favorecieron o limitaron la sobrevenida y la persistencia de trastornos musculo-esqueléticos (TME) entre operadores de un grupo industrial aeronáutico, con un enfoque diacrónico de los hechos estudiados. Un observatorio, fundado en la recopilación de datos cuantitativos sobre los operadores (dispositivo Evrest), permite relacionar los factores de riesgo TME pasados y actuales de estos operadores con su salud osteo-articular. A partir de eso se exploran los mecanismos de regulación, de desgaste y de selección eventualmente en acción. Para estos dos últimos, el análisis se basa en el estudio de "secuencias de esfuerzo" y de "acumulación de esfuerzo", el "esfuerzo" siendo definido a partir de una combinación de cuestiones sobre las exigencias físicas, la presión temporal y las posibilidades de elegir la manera de proceder en el trabajo.

Este artigo baseia-se na parte quantitativa de uma investigação em ergonomia cujo objetivo é compreender como modificações no trabalho favoreceram ou limitaram a ocorrência e persistência de lesões músculo-esqueléticas (LME) entre os operadores de um grupo industrial aeronáutico, através de uma abordagem diacrónica dos fatos estudados. Um observatório, baseado na recolha sistemática de dados quantitativos com os operadores (dispositivo Evrest), permite relacionar os fatores de risco de LME passados e atuais destes operadores com a sua saúde osteo-articular. Com base nestes dados exploram-se os mecanismos de regulação, de desgaste e de seleção eventualmente em ação. Para os dois últimos, a análise baseia-se no estudo de "sequências de esforço" e de e "acumulação de esforço", sendo o "esforço" definido a partir de um conjunto de questões sobre as exigências físicas, a pressão do tempo e as possibilidades de escolha sobre como proceder no trabalho.

Cet article se base sur la partie quantitative d'une recherche en ergonomie dont le but est de comprendre comment des évolutions du travail ont favorisé ou limité la survenue et la persistance de troubles musculo-squelettiques (TMS) chez des opérateurs d'un groupe industriel aéronautique, avec une approche diachronique des faits étudiés. Un observatoire, fondé sur le recueil systématique de données quantitatives auprès des opérateurs (dispositif Evrest), permet de mettre en relation les facteurs de risque TMS passés et présents de ces opérateurs avec leur santé ostéo-articulaire. Sur cette base, sont explorés les mécanismes de régulation, d'usure et de sélection éventuellement à l'œuvre. Pour ces deux derniers mécanismes, l'analyse repose sur 
l'étude de " séquences d'astreinte " et de " cumuls d'astreinte ", l'astreinte étant définie à partir d'une combinaison de questions sur les contraintes physiques, la pression temporelle et les possibilités de choisir la façon de procéder dans son travail.

This article is based on the quantitative part of a research in ergonomics whose goal is to understand how work modifications boosted or limited the occurrence and persistence of musculoskeletal disorders (MSD) among operators of an aeronautical industrial group, with a diachronic approach of the empirical evidence. An observatory, based on the systematic collection of quantitative data from operators (Evrest device) allows connecting operators' past and present MSD risk factors with their musculoskeletal health. On this basis, regulation, wear and selection mechanisms possibly in action are explored. For the last two mechanisms, the analysis is based on the study of "constraint sequences" and "constraint accumulation". The constraint is defined from a combination of questions about the physical constraints, the time pressure and the opportunities to choose how to proceed in work.

\section{ÍNDICE}

Mots-clés: troubles musculo-squelettiques, dispositif Evrest, régulation, usure, sélection Palabras claves: trastornos musculo-esqueléticos, dispositivo Evrest, regulación, desgaste, selección

Keywords: musculoskeletal disorders, Evrest device, regulation, wear, selection

Palavras-chave: lesões músculo-esqueléticas, dispositivo Evrest, regulação, desgaste, seleção

\section{AUTORES}

\section{CÉLINE MARDON}

Centre d'Etudes de l'Emploi, Centre de Recherches sur l'Expérience, l'Age et les Populations au Travail, Immeuble Le Descartes 1, 29 promenade Michel Simon, 93166 Noisy-le-Grand, France. celine.mardon@cee-recherche.fr

\section{WILLY BUCHMANN}

Centre d'Etudes de l'Emploi, Centre de Recherches sur l'Expérience, l'Age et les Populations au Travail, Immeuble Le Descartes 1, 29 promenade Michel Simon, 93166 Noisy-le-Grand, France. willybuchmann@aol.com

\section{SERGE VOLKOFF}

Centre d'Etudes de l'Emploi, Centre de Recherches sur l'Expérience, l'Age et les Populations au Travail, Immeuble Le Descartes 1, 29 promenade Michel Simon, 93166 Noisy-le-Grand, France. serge.volkoff@cee-recherche.fr 